\title{
Quantifying Timed-Up-and-Go Test: A Smartphone Implementation
}

\author{
Mladen Milosevic, Emil Jovanov, Aleksandar Milenković \\ Electrical and Computer Engineering Department \\ The University of Alabama in Huntsville \\ Huntsville, AL, U.S.A. \\ \{mladen.milosevic,emil.jovanov, milenka\}@uah.edu
}

\begin{abstract}
Timed-Up-and-Go (TUG) is a simple, easy to administer, and frequently used test for assessing balance and mobility in elderly and people with Parkinson's disease. An instrumented version of the test (iTUG) has been recently introduced to better quantify subject's movements during the test. The subject is typically instrumented by a dedicated device designed to capture signals from inertial sensors that are later analyzed by healthcare professionals. In this paper we introduce a smartphone application called sTUG that completely automates the iTUG test so it can be performed at home. sTUG captures the subject's movements utilizing smartphone's built-in accelerometer and gyroscope sensors, determines the beginning and the end of the test and quantifies its individual phases, and optionally uploads test descriptors into a medical database. We describe the parameters used to quantify the iTUG test and algorithms to extract the parameters from signals captured by the smartphone sensors.
\end{abstract}

Keywords-mobile computing, mobile sensing, health monitoring, timed-up-and-go test, mobility assessment, Parkinson's disease.

\section{INTRODUCTION}

The Timed-Up-and-Go (TUG) is a frequently used clinical test for assessing balance, mobility, and fall risk in elderly population and people with Parkinson's disease [1]. It is simple and easy to administer in an office, and thus can be used in screening protocols. The test measures the time a person takes to perform the following tasks: rise from a chair, walk three meters, turn around, walk back to the chair, and sit down. Longer TUG times have been associated with mobility impairments and increased fall risks [1]-[3]. Adults without balance problems can perform this test in less than 10 seconds. Alternatively, adults with mobility difficulty may require more than 30 seconds. TUG duration is also sensitive to therapeutic interventions, e.g., in Parkinson's patients [4], [5]. Whereas the test has been proven valuable in early assessment of balance and mobility, it is limited as its only outcome is the time to complete the test.

An instrumented Timed-Up-and-Go (iTUG) test has been recently introduced [6], [7]. In this test, the subject is instrumented by a dedicated device specially designed for gait and movement analysis. A number of additional parameters can be derived that can better indicate gait and balance impairments, including Sit-to-Stand duration, Standto-Sit duration, the amplitude range of anterior-posterior acceleration, etc. iTUG has proven to be sensitive to pathologies [6], [7] and useful in fall risk prediction [8].

The prior TUG studies utilized either specialized devices for movement analysis (e.g., McRoberts Dynaport Hybrid) or custom inertial sensors (e.g. accelerometers) that were mounted on the subject's lower back. Such devices typically include a 3-dimensional accelerometer and can record $\mathrm{x}, \mathrm{y}$, and $\mathrm{z}$ acceleration components during the TUG test. The data are later analyzed off-line to parameterize the TUG test. Mellone et al evaluated suitability of a smartphone's built-in accelerometer for the iTUG [9]. They compared the subject's anteroposterior acceleration measured concurrently on a smartphone and a state-of-the-art device for movement analysis, and found the statistical agreement between the two. However, this study did not pursue a more ambitious goal of using smartphone application for quantifying the TUG test.

Recognition and quantification of human activities using small wearable sensors during activities of daily living has been increasingly used in many applications. Automatic activity recognition and quantification systems that utilize inertial sensors are proposed for long-term health and fitness monitoring [10], [11], assessment of mobility in elderly and people with Parkinson's disease [12]-[14], automatic fall detection [9], [15], and rehabilitation [16], [17]. Approaches for automatic activity recognition used by researchers vary in number, type, and placement of utilized sensors, as well as in processing of recorded signals. While some researchers used multiple sensors for automatic activity recognition [18]-[20] increasing number of projects use a single inertial sensor [21]-[24] usually placed on the subject's chest.

Modern smartphones integrate a growing number of inertial and location sensors, such as an accelerometer, magnetometer (digital compass), gyroscope, and GPS. Major mobile operating systems, such as Android, iOS, and Windows 8 support frameworks for managing the sensors, including continual sampling, thus enabling a wide variety of new mobile sensing applications in different domains.

In this paper we introduce a smartphone iTUG application called $s T U G$. A subject mounts the smartphone on his/her chest or belt and starts the application. The application records and processes the signals from the smartphone's gyroscope and accelerometer sensors to extract the following parameters that quantify individual phases of the TUG test: (a) the total duration of the TUG test, (b) the 
total duration of the sit-to-stand transition, and (c) the total duration of the stand-to-sit transition. In addition, we extract parameters that further quantify body movements during the sit-to-stand and stand-to-sit transitions, including the duration of sub phases, maximum angular velocities and upper trunk angles. These parameters are recorded on the smartphone and optionally uploaded to an mHealth server.

sTUG could be of great interest for older individuals and Parkinson's disease patients as well as for healthcare professionals. The procedure requires minimum setup (chair and marked distance of 3 meters) and inexpensive instrumentation (smartphone placed on the chest or belt running the sTUG application). The feedback is provided instantaneously to the user in a form of a report with the values of all significant parameters that characterize the TUG test. It is easy to use and users can take multiple tests in a single day at home (e.g., to assess the effects of drugs). With automatic updates to the mHealth server, caregivers and healthcare professionals can gain insights into overall wellness of the subjects. For example, they can assess the impact of therapeutic interventions (e.g., impact of drugs) by analyzing the parameters from multiple tests performed in a single day. Next, healthcare professionals and researchers can monitor and evaluate evolution of disease by analyzing the trends in the parameters collected over longer periods of time.

To the best of our knowledge, we are the first to propose and implement a smartphone application that fully automates the iTUG test with minimal user interventions. We introduce parameters and procedures for their extraction that improve characterization of the iTUG test and especially body posture transitions relative to the previously proposed parameters [6].

The body instrumentation, body movements capturing, and signal processing to extract the parameters are described in Section II. The smartphone application is described in Section III. The results from preliminary tests performed on three Parkinson's disease patients and four healthy individuals are given in Section IV. Section V gives the concluding remarks.

\section{INSTRUMENTED TIMED-UP-AND-GO: FROM SIGNALS TO INFORMATION}

\section{A. Body instrumentation}

Figure 1 illustrates typical phases of the iTUG test that involves a sit-to-stand transition, walking forward for 3 meters, turning around a cone, walking back to the chair, and a stand-to-sit transition. We instrument a subject by placing a smartphone on his or her chest. The smartphone is placed in a holder that is mounted on two elastic textile straps, so it remains fixed relative to the trunk position during a test. Whereas this setup may pose challenges to some of the subjects who are unable to tie a strap over their chest without assistance, alternative attachments are possible. For example, the smartphone may be placed in a textile holder that is worn around the neck and attached to the clothes using a Velcro strap. Alternative setups are possible with a smartphone placed in a belt or in a holder on the hip. However, in this paper we assume that the smartphone is placed close to sternum in an upright position or clipped on the front side of a waist belt.

Figure 2 shows the smartphone orientation: the smartphone's $z$ axis corresponds to the sagittal axis, the $y$ corresponds to the vertical axis, and the $\mathrm{x}$ corresponds to the frontal axis of the human body.

Modern smartphones typically integrate a number of inertial and navigation sensors including an accelerometer, a gyroscope, and a magnetic sensor. The accelerometer measures proper acceleration and is typically used to keep the screen upright regardless of the smartphone orientation. With the proposed mounting of the smartphone, we record the $x, y$, and $z$ components of the acceleration of the subject's upper trunk and use these signals to determine upper body position. The gyroscope measures angular movements, specifically the rotation around the $x$ axis (a.k.a. roll), $y$ axis (yaw), and $z$ axis (pitch). In our setup, we record the angular speeds from the gyroscope and use the signals to detect and locate important transitions during the TUG test. The magnetic sensor is sensing the Earth's magnetic field and is normally used to aid navigation by determining the Earth's magnetic poles and the smartphone's orientation. In our setup we can use the signal to detect changes in direction during walking.
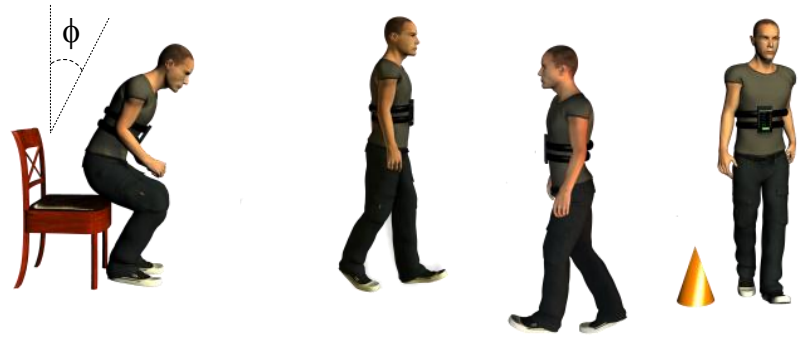

Figure 1. iTUG test phases and smartphone instrumentation of the subject.

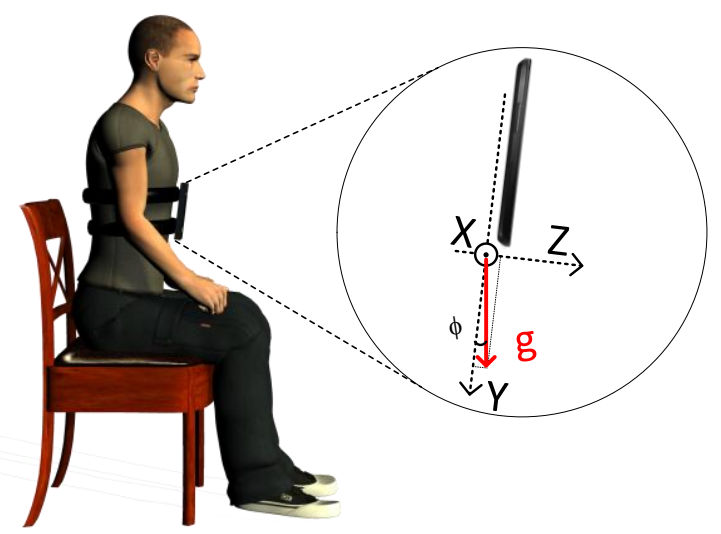

Figure 2. Smartphone orientation.

\section{B. iTUG Characterization}

To characterize the TUG test we start from a list of parameters suggested by Weiss et. al [6]. We expand this list to include new parameters and to refine the definitions of the 
original parameters that were lacking due to limitations by the measuring setup used in [6] and improper interpretations of signals. Table 1 lists the proposed parameters and describe their meaning and metric units. In addition to the total duration of the test $(d . T U G)$, we determine the total duration of the sit-to-stand transition (d.S2ST), and the total duration of the stand-to-sit transition (d.ST2S). To better characterize the sit-to-stand transition we determine its two phases - a leaning forward (d.LF) phase and lifting up phase (d.LT), the maximum trunk angle (a.S2ST) during the lean forward phase, the maximum angular velocities in the lean forward (v. $L F)$ and the lift up phase (v.LT). Similarly, we determine duration of separate phases of the stand-to-sit transition, a preparing to sit phase $(d . P S)$ and a sitting down phase (d.SD).

\section{TABLE I. PARAMETERS FOR ITUG CHARACTERIZATION.}

\begin{tabular}{|l|l|l|}
\hline $\begin{array}{c}\text { Parame- } \\
\text { ter }\end{array}$ & \multicolumn{1}{|c|}{ Description } & Units \\
\hline d.TUG & Total duration of the TUG test & seconds \\
\hline d.S2ST & Total duration of the sit-to-stand transition & seconds \\
\hline d.LF & $\begin{array}{l}\text { Duration of the lean forward phase in } \\
\text { the sit-to-stand transition }\end{array}$ & seconds \\
\hline d.LT & $\begin{array}{l}\text { Duration of the lift phase in } \\
\text { the sit-to-stand transition }\end{array}$ & seconds \\
\hline a.S2ST & $\begin{array}{l}\text { Maximum change of the trunk angle } \\
\text { in the lean forward phase }\end{array}$ & degrees/s \\
\hline v.LF & $\begin{array}{l}\text { Maximum angular velocity } \\
\text { during the lean forward phase }\end{array}$ & degrees/s \\
\hline v.LT & $\begin{array}{l}\text { Maximum angular velocity during } \\
\text { the lift up phase }\end{array}$ & seconds \\
\hline d.ST2S & Duration of the stand-to-sit transition & seconds \\
\hline d.PS & $\begin{array}{l}\text { Duration of the prepare-to-sit phase } \\
\text { in the stand-to-sit transition }\end{array}$ \\
\hline d.SD & $\begin{array}{l}\text { Duration of the sit-down phase in } \\
\text { the stand-to-sit transition }\end{array}$ \\
\hline
\end{tabular}

Figure 3 shows raw acceleration and gyroscope data recorded on a smartphone mounted on the chest of a subject with diagnosed Parkinson's disease during the TUG test. The top graph shows the $x, y$, and $z$ components of the acceleration measured in $\mathrm{m} / \mathrm{s}^{2}$, and the bottom one shows the angular velocity measured in radian/s. The data are recorded using our custom Android application running on a Nexus 4 smartphone. The sampling frequency is set to $100 \mathrm{~Hz}$. The following subsection describes algorithms that process the raw sensor data to extract the described parameters as shown in Figure 3.

\section{Parameter Extraction}

To determine the duration of the entire TUG test and its separate phases, we need to detect and timestamp the following events:

- the beginning of the test $\left(\mathrm{T}_{\mathrm{bTUG}}\right)$, which is also the beginning of the sit-to-stand transition $\left(\mathrm{T}_{\mathrm{bS} 2 \mathrm{ST}}\right)$;

- $\quad$ the end of the sit-to-stand transition $\left(\mathrm{T}_{\mathrm{eS} 2 \mathrm{ST}}\right)$;

- the start of the stand-to-sit transition $\left(\mathrm{T}_{\mathrm{bST} 2 \mathrm{~S}}\right)$; and

- the end of the stand-to-sit transition, which also corresponds to the end of the entire test $\left(\mathrm{T}_{\mathrm{eTUG}}\right)$.

The first step in detecting the beginning of the sit-tostand transition is to search for a change in the angular velocity around the frontal axis (the $x$ component of the gyroscope). This change is caused by leaning forward as the individual prepares to stand up. The angular velocity has a distinct profile as illustrated in Figure 4, left. It starts from zero or near zero, increases to reach the maximum and drops to zero at the end of the lean forward phase. To determine the beginning of the transition we first find the maximum angular velocity (v.LF) that is above a certain threshold and then search backward to find the beginning of the slope. The
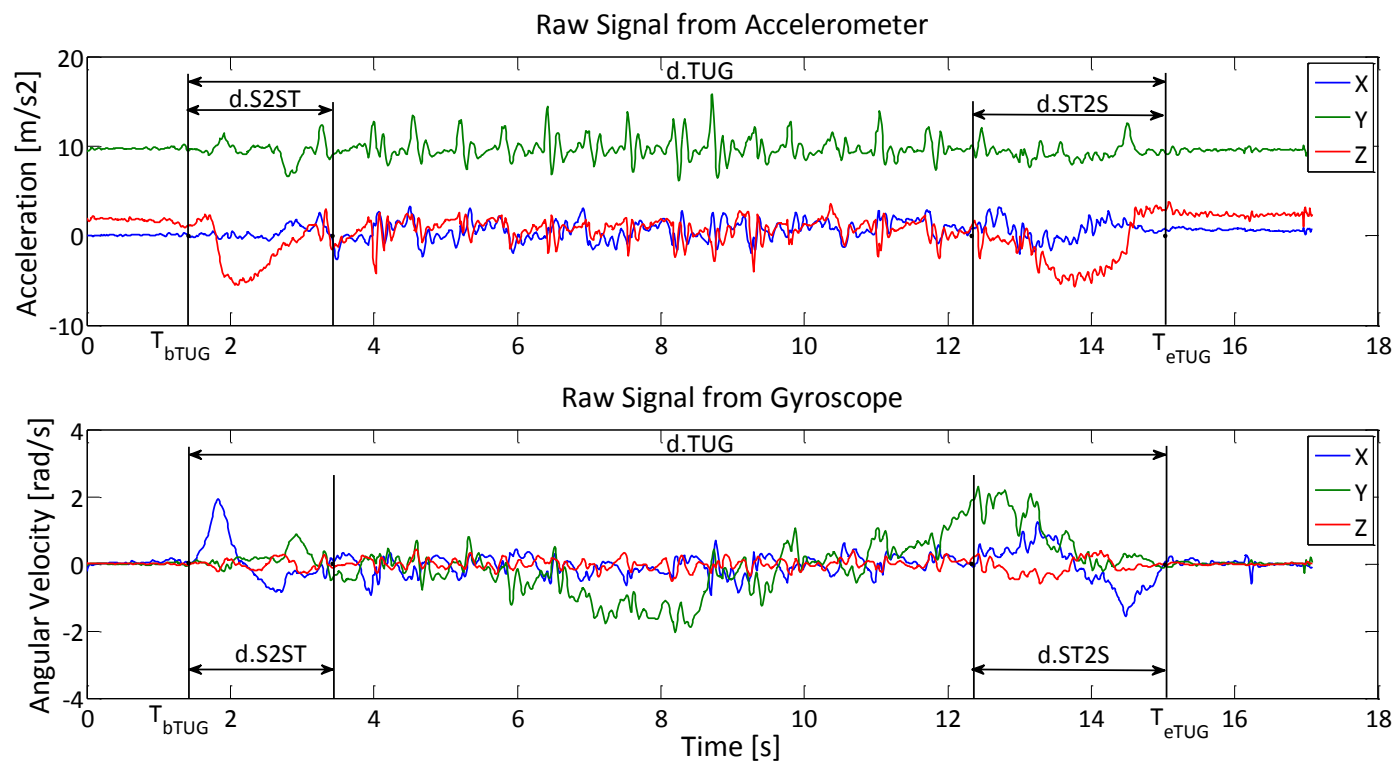

Figure 3. Accelerometer and gyroscope signals (the $\mathrm{x}, \mathrm{y}$, and $\mathrm{z}$ components) recorded on a smartphone mounted on a person with Parkinson's disease during the TUG test. 
lean forward phase ends when the angular velocity drops back to zero $\left(\mathrm{T}_{\mathrm{eLF}}\right)$. At this moment the maximum upper trunk angle, a.S2ST, is reached (Figure 4, left). The time distance between the beginning of the transition and the end of the lean forward phase represents the duration of the lean forward phase, $d . L F=T_{e L F}-T_{b S 2 S T}$. The second phase of the sit-to-stand transition is characterized by the negative angular velocity as the subject moves into the upright position. The angular velocity reaches the minimum, v.LT, and then increases back to zero. The moment when it becomes positive is considered to be the end of the lift up phase and the end of the sit-to-stand transition. By time stamping this moment $\left(\mathrm{T}_{\mathrm{eS} 2 \mathrm{ST}}\right)$, we calculate the duration of the lift up phase, d.LT $=\mathrm{T}_{\mathrm{eS} 2 \mathrm{ST}}-\mathrm{T}_{\mathrm{eLF}}$, and the total duration of the sit-to-stand transition, d.S2ST $=$ d.LF + d.LT.
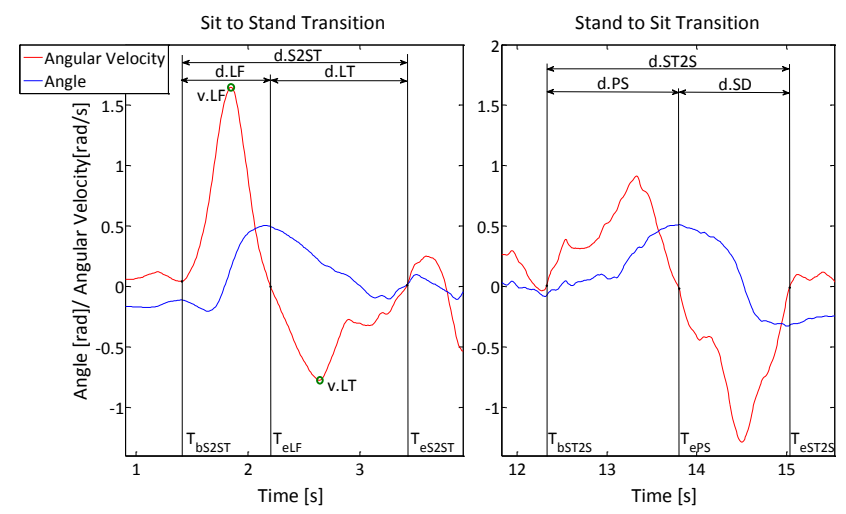

Figure 4. Angular velocity and upper body trunk angle during the sit-tostand (left) and stand-to-sit (right) transitions.

The stand-to-sit transition can also be divided into two separate phases, a prepare-to-sit, PS, and a sit-down, SD, phase. The angular velocity and the upper trunk angle profiles during the stand-to-sit transition are shown in Figure 4 , right. The angular velocity increases to the maximum and then drops back to zero as the subject leans forward in preparation to sit down. The moment when the angular velocity drops to zero marks the end of the prepare-to-sit phase and the beginning of the sit-down phase. By time stamping these characteristic points we can determine the duration of the preparation phase, d.PS $=T_{e P S}-T_{b S T 2 s}$. In the sit-down phase the angular velocity is negative as the subject's upper trunk moves back into the upright position. The moment it becomes positive marks the end of the sitdown phase and the entire stand-to-sit transition. By time stamping this moment we can calculate the duration of the sit-down phase, d.SD $=\mathrm{T}_{\mathrm{eST} 2 \mathrm{~S}}-\mathrm{T}_{\mathrm{ePS}}$, and the entire stand-tosit transition $(\mathrm{d} . \mathrm{ST} 2 \mathrm{~S}=\mathrm{d} . \mathrm{PS}+\mathrm{d} . \mathrm{SD})$.

\section{STUG}

The sTUG smartphone application captures the signals from the smartphone sensors, processes the data to extract the parameters described in Section II, and displays the parameters at the end of the TUG test. In addition it creates an iTUG test descriptor that includes time and date when the test is taken as well as all the parameters from Table I. This descriptor is stored on the smartphone. It can optionally be uploaded to a medical server for long-term storage and analysis.

The user starts monitoring and processing by pressing a start button on the screen. Alternatively, the application and monitoring can be started automatically using an inexpensive Near Field Communication (NFC) tag. The application stops monitoring automatically once it detects the end of the standto-sit transition. Figure 5 shows a report generated by the application at the end of a TUG test.

sTUG is developed for Android operating systems and requires a smartphone with the accelerometer and gyroscope sensors running Android 2.3 or above. The application has been tested on a Nexus 4 smartphone, a Motorola RAZR M, and a RAZR HD.

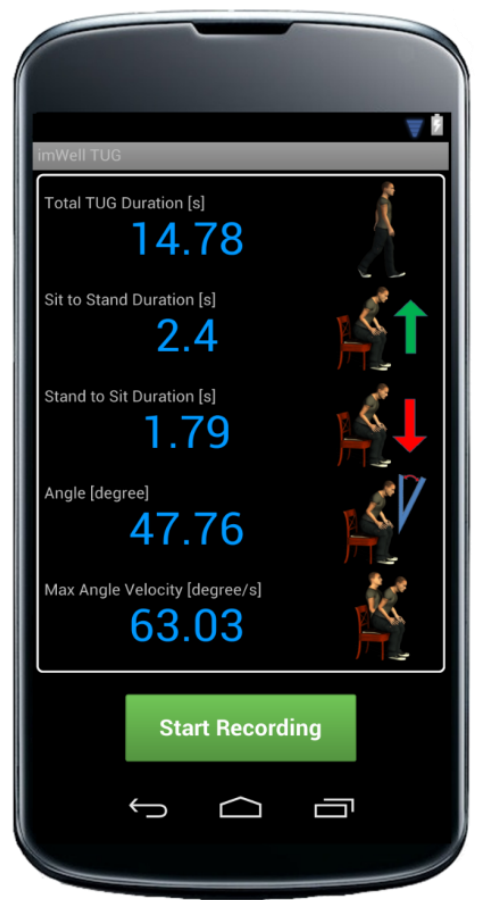

Figure 5. sTUG: Smartphone TUG Android application screen displaying the parameters of the TUG test.

\section{RESUlts AND Discussion}

A preliminary testing of the sTUG application is performed on three individuals with diagnosed Parkinson's disease and four healthy individuals. Each individual was asked to perform the TUG test in the shortest amount of time and the test is repeated three times. Table 2 shows the summarized results with all parameters reported by the sTUG application. Expectedly, the individuals with Parkinson's disease needed more time to complete the total test (d.TUG) as well as the individual phases of the test. The healthy individuals had notably higher the maximum angular velocity during the lift up phase (v.LT). The duration of the stand-to-sit phase for healthy individuals was notably shorter. 
TABLE II ITUG PARAMETERS FOR 3 INDIVIDUALS WITH DIAGNOSED PARKINSON'S DISEASE (S1-S3) AND 4 HEALTHY INDIVIDUALS (S4-S7).

\begin{tabular}{|c|c|c|c|c|c|c|c|c|c|c|}
\hline Subject & d.TUG [s] & d.S2ST [s] & d.LF [s] & d.LT [s] & a.S2ST[deg] & v.LF [deg/s] & v.LT [deg/s] & d.ST2S [s] & d.PS [s] & d.SD [s] \\
\hline \hline S1 & $11.65 \pm 0.99$ & $2.59 \pm 0.08$ & $1.27 \pm 0.18$ & $1.32 \pm 0.24$ & $52.78 \pm 4.12$ & $124.77 \pm 27.54$ & $-87.17 \pm 1.38$ & $3.15 \pm 0.53$ & $1.68 \pm 0.22$ & $1.47 \pm 0.31$ \\
\hline S2 & $11.45 \pm 0.62$ & $2.13 \pm 0.44$ & $0.99 \pm 0.15$ & $1.14 \pm 0.3$ & $55.54 \pm 10.89$ & $141.23 \pm 19.24$ & $-88.45 \pm 21.67$ & $2.28 \pm 0.03$ & $1.23 \pm 0.15$ & $1.05 \pm 0.17$ \\
\hline S3 & $14.4 \pm 0.68$ & $2.74 \pm 0.71$ & $1.55 \pm 0.73$ & $1.18 \pm 0.04$ & $44.84 \pm 10.87$ & $94.46 \pm 27.13$ & $-55.02 \pm 17.67$ & $3.02 \pm 0.33$ & $1.81 \pm 0.3$ & $1.21 \pm 0.22$ \\
\hline S4 & $7.03 \pm 0.24$ & $2.65 \pm 0.27$ & $0.98 \pm 0.13$ & $1.67 \pm 0.28$ & $56.82 \pm 15.96$ & $115.2 \pm 36.29$ & $-100.0 \pm 6.75$ & $1.51 \pm 0.04$ & $0.6 \pm 0.03$ & $0.9 \pm 0.07$ \\
\hline S5 & $4.92 \pm 0.09$ & $1.75 \pm 0.1$ & $0.7 \pm 0.03$ & $1.05 \pm 0.13$ & $76.28 \pm 1.57$ & $189.02 \pm 0.62$ & $-164.71 \pm 19.11$ & $1.31 \pm 0.03$ & $0.5 \pm 0.01$ & $0.81 \pm 0.02$ \\
\hline S6 & $5.15 \pm 0.11$ & $1.61 \pm 0.12$ & $0.73 \pm 0.10$ & $0.88 \pm 0.03$ & $71.72 \pm 4.09$ & $207.07 \pm 11.85$ & $-221.18 \pm 12.68$ & $1.65 \pm 0.07$ & $0.81 \pm 0.03$ & $0.84 \pm 0.06$ \\
\hline S7 & $6.86 \pm 0.49$ & $2.23 \pm 0.17$ & $0.97 \pm 0.11$ & $1.26 \pm 0.23$ & $59.43 \pm 9.39$ & $146.41 \pm 18.22$ & $-126.26 \pm 24.56$ & $1.5 \pm 0.32$ & $0.7 \pm 0.18$ & $0.8 \pm 0.24$ \\
\hline
\end{tabular}

[4] E. Auriel, J. M. Hausdorff, T. Herman, E. S. Simon, and N. Giladi, "Effects of methylphenidate on cognitive function and gait in patients with Parkinson's disease: a pilot study," Clin Neuropharmacol, vol. 29, no. 1, pp. 15-17, Feb. 2006.

\section{CONCLUSIONS}

Proliferation of smartphones that integrate a growing number of sophisticated sensors creates a number of opportunities for instrumentation and quantification of standard monitoring and diagnostic procedures. The TimedUp-and-Go test is frequently used to assess mobility and balance of elderly and people with Parkinson's disease. In this paper we introduce a smartphone application called sTUG that completely automates the test. The application provides an instantaneous feedback to the user and allows for automatic uploads of the test results into patient medical record. The application quantifies the test phases to allow specialists better assessment of body kinematics and dynamics.

The application has been tested on a group of healthy volunteers and Parkinson's disease patients and showed promising results. By utilizing commodity smartphones we offer an affordable tool for instantaneous quantification of the TUG tests. The test can thus be performed at home to assess the impact of drugs. Analysis of the parameters collected over longer periods of time may help in tracking progression of mobility and balance impairments.

\section{ACKNOWLEDGMENT}

This work was supported in part by U.S. National Science Foundation under grant CNS-1205439. We would like to thank the Parkinson's Support Group of Huntsville for their help in conducting the tests.

\section{REFERENCES}

[1] D. Podsiadlo and S. Richardson, "The timed 'Up \& Go': a test of basic functional mobility for frail elderly persons," J Am Geriatr Soc, vol. 39, no. 2, pp. 142-148, Feb. 1991.

[2] B. Najafi, K. Aminian, F. Loew, Y. Blanc, and P. A. Robert, "Measurement of stand-sit and sit-stand transitions using a miniature gyroscope and its application in fall risk evaluation in the elderly," IEEE Trans Biomed Eng, vol. 49, no. 8, pp. 843-851, Aug. 2002.

[3] "Guideline for the prevention of falls in older persons. American Geriatrics Society, British Geriatrics Society, and American Academy of Orthopaedic Surgeons Panel on Falls Prevention," $J$ Am Geriatr Soc, vol. 49, no. 5, pp. 664-672, May 2001.
[5] R. Ben-Itzhak, N. Giladi, L. Gruendlinger, and J. M. Hausdorff, "Can Methylphenidate Reduce Fall Risk in Community-Living Older Adults? A Double-Blind, Single-Dose Cross-Over Study," Journal of the American Geriatrics Society, vol. 56, no. 4, pp. 695 700, Apr. 2008.

[6] A. Weiss, T. Herman, M. Plotnik, M. Brozgol, I. Maidan, N. Giladi, T. Gurevich, and J. M. Hausdorff, "Can an accelerometer enhance the utility of the Timed Up \& Go Test when evaluating patients with Parkinson's disease?," Med Eng Phys, vol. 32, no. 2, pp. 119-125, Mar. 2010.

[7] C. Zampieri, A. Salarian, P. Carlson-Kuhta, K. Aminian, J. G. Nutt, and F. B. Horak, "The instrumented timed up and go test: potential outcome measure for disease modifying therapies in Parkinson's disease," Journal of Neurology, Neurosurgery \& Psychiatry, vol. 81, no. 2, pp. 171-176, Sep. 2009.

[8] M. Marschollek, G. Nemitz, M. Gietzelt, K. H. Wolf, H. Meyer zu Schwabedissen, and R. Haux, "Predicting in-patient falls in a geriatric clinic," Zeitschrift für Gerontologie und Geriatrie, vol. 42, no. 4, pp. 317-322, Jun. 2009.

[9] C. Tacconi, S. Mellone, and L. Chiari, "Smartphone-based applications for investigating falls and mobility," in 20115 th International Conference on Pervasive Computing Technologies for Healthcare (PervasiveHealth), 2011, pp. 258 -261.

[10] S. Bosch, M. Marin-Perianu, R. Marin-Perianu, P. Havinga, and H. Hermens, "Keep on Moving! Activity Monitoring and Stimulation Using Wireless Sensor Networks," in Smart Sensing and Context, P. Barnaghi, K. Moessner, M. Presser, and S. Meissner, Eds. Springer Berlin Heidelberg, 2009, pp. 11-23.

[11] F. Pitta, T. Troosters, V. S. Probst, M. A. Spruit, M. Decramer, and R. Gosselink, "Quantifying physical activity in daily life with questionnaires and motion sensors in COPD," Eur. Respir. J., vol. 27, no. 5, pp. 1040-1055, May 2006.

[12] R. Ganea, A. Paraschiv-Ionescu, C. Büla, S. Rochat, and K. Aminian, "Multi-parametric evaluation of sit-to-stand and stand-tosit transitions in elderly people," Medical Engineering \& Physics, vol. 33, no. 9, pp. 1086-1093, Nov. 2011.

[13] A. Al-Jawad, M. R. Adame, M. Romanovas, M. Hobert, W. Maetzler, M. Traechtler, K. Moeller, and Y. Manoli, "Using multidimensional dynamic time warping for TUG test instrumentation with inertial sensors," in 2012 IEEE Conference on Multisensor Fusion and Integration for Intelligent Systems (MFI), 2012, pp. 212 -218 .

[14] A. Zijlstra, M. Mancini, U. Lindemann, L. Chiari, and W. Zijlstra, "Sit-stand and stand-sit transitions in older adults and patients with Parkinson's disease: event detection based on motion sensors versus force plates," Journal of NeuroEngineering and Rehabilitation, vol. 9, no. 1, p. 75, Oct. 2012. 
[15] K. Wongpatikaseree, A. O. Lim, Y. Tan, and H. Kanai, "Rangebased algorithm for posture classification and fall-down detection in smart homecare system," in 2012 IEEE 1st Global Conference on Consumer Electronics (GCCE), 2012, pp. 243 -247.

[16] E. Jovanov, A. Milenkovic, C. Otto, and P. C. De Groen, "A wireless body area network of intelligent motion sensors for computer assisted physical rehabilitation," Journal of neuroengineering and rehabilitation, vol. 2, no. 1, p. 6, Mar. 2005.

[17] B. K. Santhiranayagam, D. T. H. Lai, C. Jiang, A. Shilton, and R. Begg, "Automatic detection of different walking conditions using inertial sensor data," in The 2012 International Joint Conference on Neural Networks (IJCNN), 2012, pp. 1 -6.

[18] L. Bao and S. S. Intille, "Activity recognition from user-annotated acceleration data," 2004, pp. 1-17.

[19] H. Ghasemzadeh, V. Loseu, and R. Jafari, "Structural Action Recognition in Body Sensor Networks: Distributed Classification Based on String Matching," IEEE Transactions on Information Technology in Biomedicine, vol. 14, no. 2, pp. 425 -435, Mar. 2010.
[20] J. Lester, T. Choudhury, and G. Borriello, "A Practical Approach to Recognizing Physical Activities," in In Proc. of Pervasive, 2006, pp. 1-16.

[21] B. Najafi and K. Aminian, "Body movement monitoring system and method," U.S. Patent EP119513910-Apr-2002.

[22] B. Florentino-Liano, N. O'Mahony, and A. Artes-Rodriguez, "Human activity recognition using inertial sensors with invariance to sensor orientation," in 2012 3rd International Workshop on Cognitive Information Processing (CIP), 2012, pp. 1-6.

[23] D. Fuentes, L. Gonzalez-Abril, C. Angulo, and J. A. Ortega, "Online motion recognition using an accelerometer in a mobile device," Expert Systems with Applications, vol. 39, no. 3, pp. 24612465, Feb. 2012.

[24] A. Godfrey, A. K. Bourke, G. M. Ólaighin, P. van de Ven, and J. Nelson, "Activity classification using a single chest mounted triaxial accelerometer," Medical Engineering \& Physics, vol. 33, no. 9, pp. 1127-1135, Nov. 2011. 